\title{
Effects of food on the central and peripheral haemodynamic response to upright exercise in normal volunteers
}

\author{
J J Yi, L Fullwood, K Stainer, A J Cowley, J R Hampton
}

\begin{abstract}
The central and peripheral haemodynamic effects of a modest meal were investigated in healthy volunteers at rest and in response to submaximal exercise. The meal increased heart rate, cardiac output, oxygen consumption, carbon dioxide production, and minute ventilation at rest and during exercise. The effects of food were additive to those induced by the exercise. Food had no effect on limb blood flow and lowered total systemic vascular resistance suggesting that there were no compensatory changes in regional blood flow to help redirect blood to the gut.

An increase in cardiac output, and therefore myocardial work, is the predominant cardiovascular response to eating and this may help explain the postprandial deterioration in symptoms of some patients with cardiovascular disorders.
\end{abstract}

The distribution of cardiac output to the main vascular beds of humans is unequal; at rest $25 \%$ is delivered to the splanchnic bed, $20 \%$ to skeletal muscle and the kidneys, $12 \%$ to the brain, $9 \%$ to the skin, and $4 \%$ to the heart. The increased demand of metabolically active tissue in response to emotional or physical stress can be met by an increase in cardiac output, a redirection of regional blood flow, or by both these mechanisms. The changes in regional blood flow are likely to be complex but are often predictable. In response to upright exercise cardiac output increases to augment blood flow to the exercising skeletal muscle of the calf and blood is redirected away from the non-exercising forearm further to help this increase in calf blood flow. ${ }^{2}$ Knowledge of these complex changes is important for our understanding of how exercise may aggravate the symptoms of patients with cardiovascular disease.

Eating is another common but less well recognised cause of stress. The first report that food increases cardiac output came in $1929^{3}$ but it was surprisingly difficult to reproduce this observation. $^{4-6}$ Intuitively, you might expect that the increased requirement for gut blood flow after a meal would be partly met by an increase in cardiac output and partly by a redistribution of blood flow from other vascular beds, but surprisingly little is known about the changes in regional blood flow after food.
Because eating can also worsen the symptoms of patients with certain cardiovascular diseases, particularly angina, it is important to examine its effects on cardiac output and regional blood flow in association with exercise.

\section{Patients and methods}

VOLUNTEERS

Ten (eight men) healthy volunteers took part in the study. They were aged $20-41$ years. All denied any symptoms suggestive of cardiovascular disease and all had a normal physical examination. All gave informed consent and the study fulfilled the requirements of the local ethics committee.

\section{METHODS}

All measurements were carried out in a temperature controlled room of $24-25^{\circ} \mathrm{C}$. The volunteers were familiarised with the experimental techniques on at least one occasion before the study.

They fasted from 12 midnight the day before the study. All rested in the laboratory for at least 30 minutes on the morning of the study before cardiac output and limb blood flow at rest were measured. The exercise test was then performed and cardiac output and oxygen consumption were measured. Immediately after the end of exercise measurements of limb blood flow were repeated.

Thirty minutes after the first exercise test the volunteers were given a standard meal of $1400 \mathrm{kcal}(5 \cdot 88 \mathrm{MJ})$ and 30 minutes after this the measurements of cardiac output and limb blood flow at rest were repeated. We have previously shown that 60 minutes' rest is sufficient time for values to return to normal. Volunteers then performed a second exercise test with measurements being made in the same way as in the first test.

\section{EXERCISE TESTING}

This was performed with a modified Bruce protocol. The speed and slope of the treadmill were increased after three minutes of stages II and IV and four minutes of the other stages to allow sufficient time for cardiac output to be measured.

$\begin{array}{llllll}\text { Stage } & \text { I } & \text { II } & \text { III } & \text { IV } & \text { V } \\ \text { Speed }(\mathrm{km} / \mathrm{h}) & 2 \cdot 7 & 2 \cdot 7 & 2 \cdot 7 & 2 \cdot 7 & 4 \cdot 0 \\ \text { Slope }^{\mathrm{o}} & 0 & 1 \cdot 3 & 2 \cdot 6 & 4 \cdot 3 & 5 \cdot 4\end{array}$

An electrocardiogram was monitored continuously and arterial blood pressure was measured at rest and during exercise with a 
Figure 1 Mean (SEM) values of heart rate at rest and during exercise in fasting volunteers and after the meal $(p<0.0001)$.

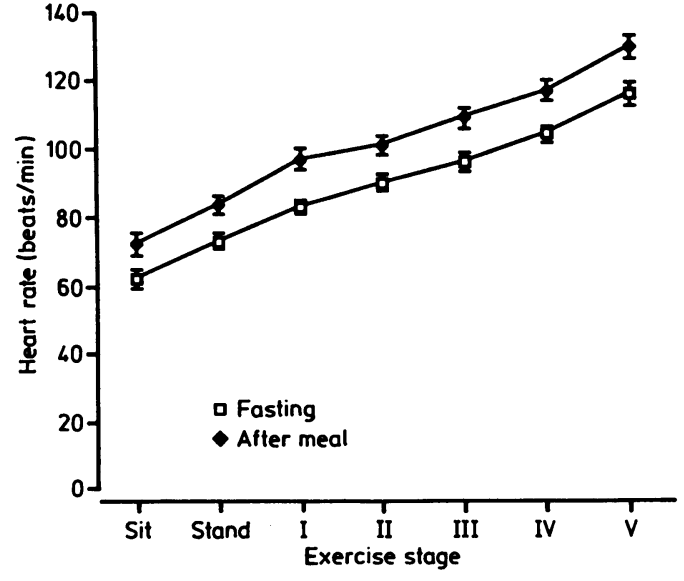

sphygmomanometer (diastolic pressure taken at phase $\mathrm{V}$ ).

\section{MEASUREMENTS OF CARDIAC OUTPUT}

Cardiac output was measured by the indirect Fick principle with carbon dioxide. ${ }^{5}$ Gas analysis was made with a mass spectrometer (VG Medical Systems). Carbon dioxide production was calculated from minute ventilation and mixed expired carbon dioxide concentration. Pulmonary venous carbon dioxide partial pressure (that is systemic arterial carbon dioxide partial pressure) was derived from end tidal carbon dioxide partial pressure, and mixed venous carbon dioxide partial pressure was estimated from a rebreathing manoeuvre. Volunteers hyperventilated into an anaesthetic bag containing a mixture of oxygen and 8-10\% carbon dioxide. When a carbon dioxide concentration, measured at the mouth, reached a plateau there was no net exchange of carbon dioxide across the lungs and this plateau value is related to mixed venous carbon dioxide partial pressure. These variables can then be used in the Fick equation to calculate cardiac output. Cardiac output was measured sitting and standing and at the end of stages I, III, and $\mathrm{V}$ of the treadmill protocol. Systemic vascular resistance was calculated by dividing mean arterial blood pressure $(2 / 3$ diastolic $+1 / 3$ systolic) by cardiac output.

\section{MEASUREMENT OF OXYGEN CONSUMPTION}

Systemic oxygen consumption was calculated from minute ventilation and the difference between mixed expired oxygen concentration and atmospheric oxygen. Measurements were made at the same times as cardiac output.

Figure 2 Mean (SEM) values of (a) systolic and (b) diastolic blood pressure at rest and during exercise in fasting volunteers and after the meal $(p=0.1442$ for systolic and $p=0.1169$ for diastolic pressure).

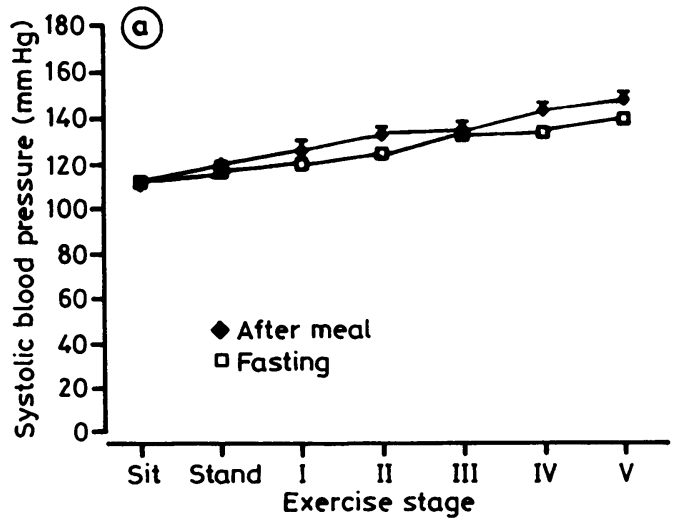

MEASUREMENT OF LIMB BLOOD FLOW

Forearm and calf blood flow were measured simultaneously by venous occlusion plethysmography with mercury in silastic strain gauges. ${ }^{8}$ Measurements were made at rest before each exercise test and from 3-13 minutes after the end of each test. ${ }^{7}$

STATISTICAL ANALYSIS

This was performed by analysis of variance for repeated measures.

\section{Results}

HEART RATE AND BLOOD PRESSURE

Figure 1 shows the effects of the meal on mean (SEM) values of heart rate at rest and during exercise. The meal increased heart rate measured with the volunteers sitting from 62 $(2 \cdot 76)$ to 72 (3.31) beats/minute and this level of increase was maintained throughout exercise $(p<0.0001)$. At the end of stage $V$ the preprandial heart rate was $116(3.40)$ compared with a value of $130(3.42)$ beats/minute after the meal.

Figure 2 shows the effect of food on mean (SEM) values of systolic and diastolic blood pressure at rest and during exercise. There was a trend to a greater increase in systolic blood pressure during exercise and a fall in diastolic pressure, but neither of these reached statistical significance $(p=0.1442$ for systolic pressure and $p=0.1169$ for diastolic blood pressure).

\section{CARDIAC OUTPUT}

Figure 3 shows the effect of food on cardiac output at rest and on the changes in cardiac output induced by exercise. Cardiac output measured when the volunteers were sitting increased from a mean (SEM) fasting value of $4.45(0.18)$ to $5.72(0.22) 1 / \mathrm{min}$ after the meal and at the end of stage $\mathrm{V}$ the fasting value was $12.70(0.60)$ compared with a postprandial value of $14.10(0.64) 1 / \mathrm{min}(p<0.0001)$.

OXYGEN CONSUMPTION, CARBON DIOXIDE PRODUCTION, AND MINUTE VENTILATION Figure 4a shows the mean (SEM) values of systemic oxygen consumption at rest and during exercise before and after the meal. Oxygen consumption in the volunteers sitting was $3.52(0.15) \mathrm{ml} / \mathrm{kg} / \mathrm{minute}$ and increased to $18.06(0.69) \mathrm{ml} / \mathrm{kg} /$ minute at stage $\mathrm{V}$ of the protocol with the subjects fasting. After the

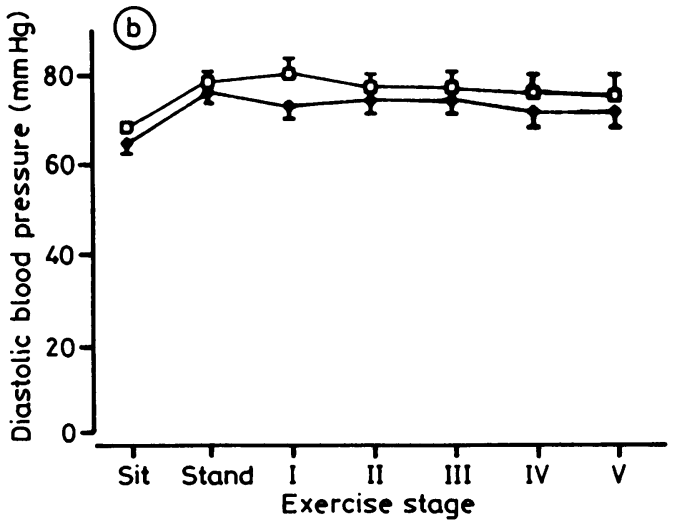



values of cardiac output at rest and during exercise in fasting volunteers and after the meal $(p<0.0001)$.

Figure 4 (a) Mean (SEM) values of systemic oxygen consumption at rest and during exercise in fasting volunteers and after the meal

$(p=0.0041)$. (b) Mean (SEM) values of minute ventilation at rest and during exercise in fasting volunteers and after the meal $(p=0.0002)$.
Figure 3 Mean (SEM)

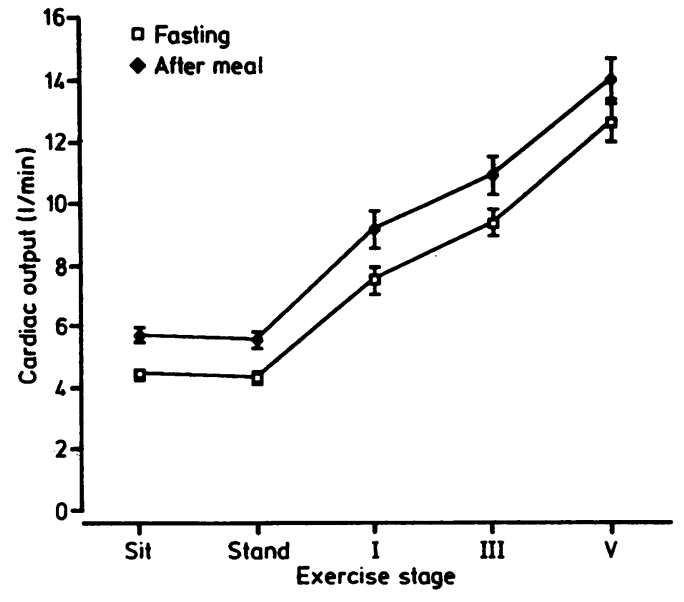

meal oxygen consumption increased significantly ( $\mathrm{p}=0.0041$ ); the value when sitting was $4.48(0.26) \mathrm{ml} / \mathrm{kg} / \mathrm{minute}$ increasing to $18.84(0.59) \mathrm{ml} / \mathrm{kg} /$ minute at stage $\mathrm{V}$ of the exercise protocol.

In association with this, carbon dioxide production was also increased postprandially $(\mathrm{p}<0.001)$. The mean value before the meal in sitting volunteers was $2.93(0.31) \mathrm{ml} / \mathrm{kg} / \mathrm{min}$; this increased to $15.91 \mathrm{ml} / \mathrm{kg} / \mathrm{min}$ at stage $\mathrm{V}$ of the protocol and after the meal the sitting value had increased to $4.04(0.25)$ and the value at stage $\mathrm{V}$ of the exercise protocol to $17.59(0.50)$ $\mathrm{ml} / \mathrm{kg} / \mathrm{min}$.

Figure $4 \mathrm{~b}$ shows that the meal increased minute ventilation at rest and during exercise $(p=0.0002)$. Mean values of minute ventilation at rest and during stage $\mathrm{V}$ of the protocol min preprandially to $8.61(0.43)$ and $26.82(1.0)$ $1 /$ min after food. increased from 6.51 (0.40) and $23.69(1.07) 1 /$

\section{LIMB BLOOD FLOW}

Figure 5 shows the changes in forearm and calf blood flow at rest and 3-13 minutes after exercise. Exercise caused the expected increase in calf blood flow and decrease in forearm blood flow. Resting limb blood flow was increased by food-in the calf from $3.34(0.48)$ to $3.71(0.37) \mathrm{ml} / 100 \mathrm{ml} / \mathrm{minute}$ and in the forearm from $2.97(0.24)$ to $3.38(0.23) \mathrm{ml} / 100$ $\mathrm{ml} / \mathrm{min}$. Although the meal had no significant effect on blood flow after exercise ( $p=0.22$ for calf and $p=0.20$ for forearm) it was always slightly higher postprandially.

SYSTEMIC VASCULAR RESISTANCE

Figure 6 shows the changes in systemic vascular resistance at rest and during exercise.

Mean systemic vascular resistance in the volunteers when sitting was lower after a meal $(14.09(0.41)$ units) than when the volunteers were fasting (18.79 (0.55) units). Throughout exercise total systemic vascular resistance was also lower after eating $(p<0.001)$.

\section{Discussion}

We found that a modest meal had considerable effects on the circulation and on the metabolic requirements of the body as measured by respiratory gas exchange. Furthermore, in response to exercise these changes persist and are additional to those produced by exercise. The changes are likely to be interrelated and may help to explain why some cardiovascular diseases are aggravated by eating.

The digestion and absorption of food is a metabolically active process that requires an increased delivery of oxygen to the gut. Eating a meal is undoubtedly associated with a higher

Figure 5 Mean (SEM) values of (a). forearm and (b) calf blood flow at rest and 3-13 minutes after exercise in fasting volunteers and after the meal ( $p=0.20$ for

forearm and $p=0.22$ for calf blood flow).
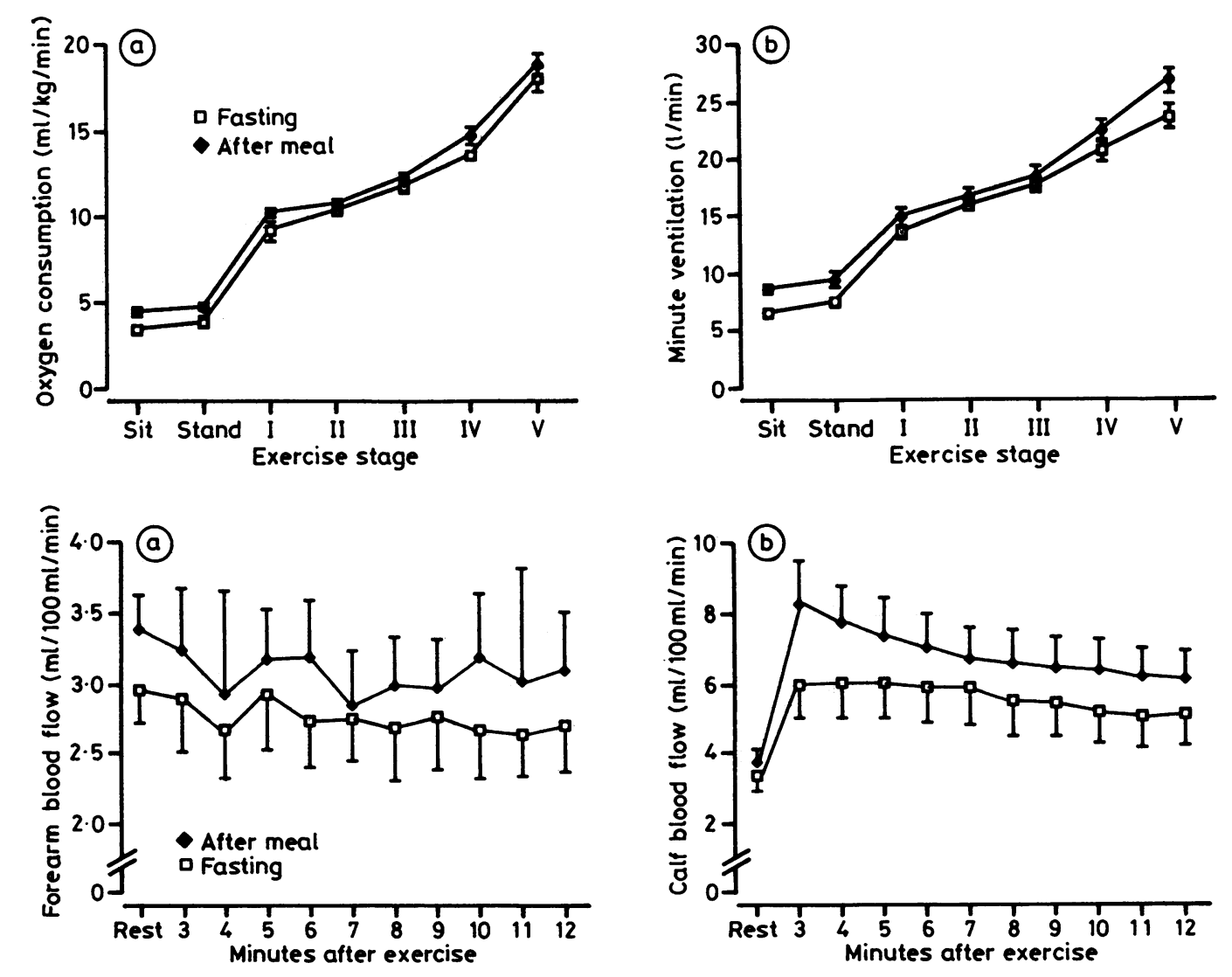
Figure 6 Mean (SEM) values for systemic vascular resistance at rest and during exercise in fasting volunteers and after the meal $(p<0.001)$.

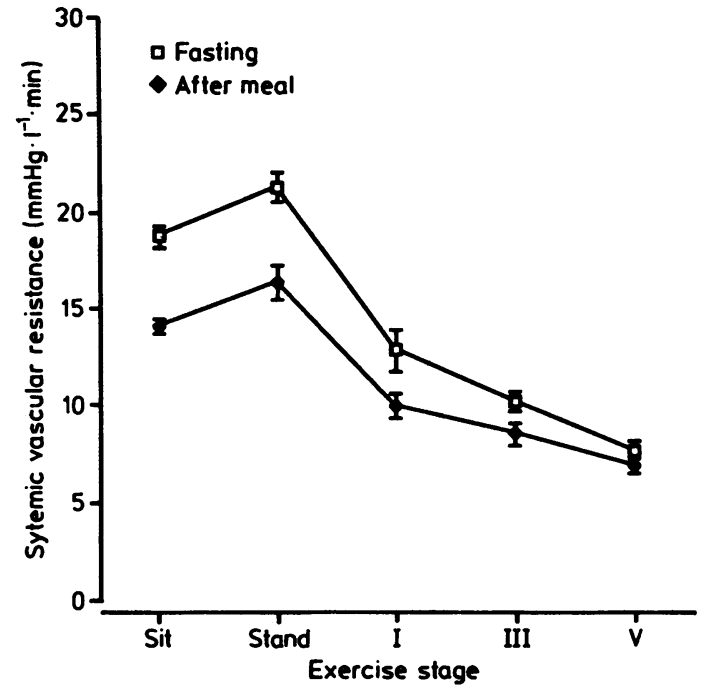

blood flow to the gut ${ }^{9}$ but precisely how this is achieved is not clear. Cardiac output could increase or blood flow could be directed away from other vascular beds. In this study, resting cardiac output increased but this was not accompanied by a reduction in blood flow to the limbs. Also, because total systemic vascular resistance fell it is unlikely that blood was directed away from other vascular beds by local vasoconstriction. Therefore, the increased blood flow requirement of the gut after food is met at rest by an increase in cardiac output and there is no evidence of redirection of blood flow from other vascular beds.

The changes during exercise are, surprisingly, similar and suggest that the cardiovascular effects of food are additive to those of exercise. We might have expected that the demand of exercising skeletal muscle would have been greater than that of the gut and that the increased blood flow to the gut after food would have been reduced during exercise to enhance delivery of blood to the calf. But this redirection did not occur, at least during submaximal exercise, and this imposes a greater workload on the myocardium to maintain a higher cardiac output.

The mechanism responsible for these changes is not absolutely clear. It has been suggested that they may be caused by increased activity of the sympathetic nervous system ${ }^{10}$ and this would certainly fit with the changes in blood pressure and heart rate that we and others have reported. ${ }^{11}$ Whatever the mechanism eating is a stressful activity and monitoring of respiratory gases indicates that it imposes an increased metabolic demand on the body. The increased oxygen consumption we have shown is not inconsiderable and matches the changes in cardiac output. The greater carbon dioxide production and minute ventilation also corresponds to the haemodynamic changes and this implies that the metabolic demands of the gut in response to food are the cause of the cardiovascular changes.

In view of the changes we have described it is surprising that more patients with cardiovascular diseases such as angina or heart failure, whose symptoms are caused either by increased myocardial work or a limited ability to increase cardiac output, do not describe a deterioration of their symptoms after a meal.

1 Mason DT, Zelis R, Longhurst J, Lee G. Cardiocirculatory response to muscular exercise in congestive heart failure. response to muscular exercise in conges

2 Cowley AJ, Stainer K, Rowley JM, Hampton JR. Abnormalities of the peripheral circulation and respiratory function in patients with severe heart failure. $\mathrm{Br}$ Heart $\mathrm{J}$ 1986;55:75-80.

3 Grollman A. Physiological variations in cardiac output III. The effect of ingestion of food on cardiac output, pulse rate, blood pressure and oxygen consumption in man. $A m$ J Physiol 1929;89:366-70.

4 Jones WB, Thomas HD, Reeves JJ. Circulatory and ventilatory response to postprandial exercise. Am Heart 1965;69:668-76.

5 Cowley AJ, Stainer K, Murphy DT, Murphy J, Hampton JR. A non-invasive method for measuring cardiac output: the effect of Christmas lunch. Lancet 1986;ii:1422-3.

6 Kelbaek H, Munck O, Christensen NJ, Godtfredsen J. Central haemodynamic changes after a meal. Br Heart 1989;61:506-9.

7 Cowley AJ, Rowley JM, Stainer K, Hampton JR. Effects of captopril on abnormalities of the peripheral circulation and respiratory function in pati

8 Greenfield ADM, Whitney RI, Mowbray FR Methods for the investigation of peripheral blood flow. Br Med Bull the investigation

9 Qamar MI, Read AE, Skidmore R, Evans JM, Wells PNT Transcutaneous doppler ultrasound measurement of superior mesenteric artery blood flow in man. Gut 1986 27:100-5

10 Kelbaek H, Munck O, Christensen NJ, Godtfredsen J Autonomic nervous control of postprandial hemodynamic changes at rest and upright exercise. $J$ Appl Physio 1987;63:1862-5.

11 De Mey C, Hansen-Schmidt S, Enterling D. Postprandial haemodynamic changes: a source of bias in cardiovascular research affected by its own methodological bias. Cardiovasc Res 1988;22:703-7. 